\title{
A Compact Model of Backscattering Coefficient and Mobility of Graphene FET for SiO2 and h-BN Substrates
}

Abhishek Kumar Upadhyay ( $\square$ meetabhishek14@gmail.com )

X-Fab (Germany) https://orcid.org/0000-0003-3901-201X

\section{Deepika Gupta}

IIITDM Kancheepuram: Indian Institute of Information Technology Design and Manufacturing

Kancheepuram

\section{Ribu Mathew}

VIT Bhopal University

Ankur Beohar

VIT Bhopal University

\section{Research Article}

Keywords: Scattering rate, backscattering coefficient, mobility, GFET

Posted Date: January 18th, 2022

DOI: https://doi.org/10.21203/rs.3.rs-1245210/v1

License: (c) (i) This work is licensed under a Creative Commons Attribution 4.0 International License. Read Full License 


\title{
A Compact Model of Backscattering Coefficient and Mobility of Graphene FET for $\mathrm{SiO}_{2}$ and h-BN Substrates
}

\author{
Abhishek Kumar Upadhyay ${ }^{1 *}$, Deepika Gupta², Ribu \\ Mathew $^{3}$ and Ankur Beohar ${ }^{3}$ \\ $1^{*}$ Chair for Electron Devices and Integrated Circuits, Technical \\ University Dresden, Helmholtzstrasse 18, City, 01069, Dresden, \\ Germany. \\ ${ }^{2}$ Department of ECE, IIIT Naya Raipur, Sector 24, Naya Raipur, \\ 493661, Chhattisgarh, India. \\ ${ }^{3}$ Department of ECE, VIT Bhopal, Street, Bhopal, 610101, M.P., \\ India.
}

\footnotetext{
*Corresponding author(s). E-mail(s): meetabhishek14@gmail.com;
}

\begin{abstract}
The field dependent compact model of backscattering coefficient and quasi-ballistic mobility of charge carriers in graphene have developed for two different substrates: silicon dioxide $\left(\mathrm{SiO}_{2}\right)$, and hexagonal boron nitride (h-BN). The formulation of the backscattering coefficient is performed using the Landauer and McKelvey Flux Theory (MFT) in quasi-ballistic regime. In graphene, acoustic phonon (AP), surface optical phonon (OP) and charged impurity (CI) scatterings are present among the charge carriers. That is carefully considered during the formulation of backscattering coefficient (R) and quasiballistic mobility $\left(\boldsymbol{\mu}_{\boldsymbol{e f f}}\right)$. We find that the Graphene Field Effect Transistor (GFET) with h-BN substrate has the lower backscattering and higher quasi-ballistic mobility. The modeled expression of backscattering coefficient and quasi-ballistic mobility is substituted in drain current $\left(\boldsymbol{I}_{\boldsymbol{D} S}\right)$ of GFET devices having $\mathrm{SiO}_{2}$ and h-BN substrate, have shown good agreement with the experimental results.
\end{abstract}

Keywords: Scattering rate, backscattering coefficient, mobility, GFET 


\section{Introduction}

Graphene monolayer, two-dimensional (2D) atomic thick sheet of carbon atoms arranged in a honeycomb lattice. Due to the exotic electrical and mechanical properties, graphene is being extensively explored as an alternative channel material for Metal Oxide Semiconductor (MOS) devices. It has ultra-high intrinsic carrier mobility, and saturation velocity, which makes it compatible for radio frequency $(\mathrm{RF})$ applications, but its gapless nature makes it unfit for digital applications [1]-[4]. The high carrier transport may lead the carrier conduction in the quasi-ballistic regime, which is strongly affected by optical phonon (OP), charged impurity (CI) and acoustic phonon (AP) scattering rates scattering mechanism during the moving from source to drain in graphene field effect transistor (GFET) [5],[6]. Among these scattering mechanisms, OP and CI scatterings are induce by the substrate of the GFET device. A reliable substrate is required for the GFET device to achieve the high-performance.

Over the past few years, several studies have been made by different research groups, to portray the different scattering mechanism induced by the substrate of the GFET device [7]-[12]. The $\mathrm{SiO}_{2}$, and hexagonal BN (h$\mathrm{BN}$ ), are the commonly used substrates. The $\mathrm{SiO}_{2}$ substrate is used due to its conventional technology compatibility, but it has offer additional scattering mechanisms [13],[14]. The impact of these scattering mechanism is on the device performance is measured in terms of the field dependant Backscattering coefficient $(\mathrm{R})$ and quasi-ballistic mobility [15]. However, the quasi-ballistic mobility for $\mathrm{SiO}_{2}$ substrate is strongly affected by the additional graphene/substrate interface charge impurities, optical phonons (OPs), and surface roughness scattering mechanisms [5]. The hexagonal boron nitride (h-BN) is used as a substrate for GFET device to overcome with these scattering mechanisms. The h-BN is an atomically smooth surface with minimal dangling bonds, and lower charge traps at graphene/substrate interface $\left(2.5 \times 10^{10} \mathrm{~cm}^{-2}\right)$. Its lattice constant is also similar to that of graphene, and having large optical phonon energy and bandgap [8],[10],[16].

Motivated from this, we develop an analytical model of field dependant backscattering coefficient expression, and quasi-ballistic mobility to theoretically investigate the effect of different substrate induced scattering mechanisms. To formulate the backscattering coefficient the one dimensional (1D) Boltzmann transport equation (BTE) is solved using two dimensional (2D) relaxation length approximations $\left(\lambda_{2 D}\right)$. This equation will further solved with $1 \mathrm{D}$ linear potential energy profile, which is leading to an analytical expression for the backscattering coefficient [17]-[19]. The developed expression is valid of low- and high-electric fields (i.e., short and long channel) GFET devices. The quasi-ballistic mobility is expressed with the help of the average carrier energy, transmission coefficient $(\mathrm{T})$, number of transmission channel $\left(N_{T}(E)\right)$, and two dimensional relaxation length approximation. However, during the formulation of $\lambda_{2 D}$, different scattering mechanisms are carefully considered because $\lambda_{2 D}$ is strongly affected by these different scattering mechanisms induced by the substrate of GFET device [20]. 
Table 1: Different Parameters and its Values, which are used in the Modeled Expressions of Backscattering Coefficient, Quasi-Ballistic Mobility and Drain Current

\begin{tabular}{|c|c|c|c|c|}
\hline \multirow[b]{2}{*}{ Parameters } & \multicolumn{2}{|c|}{ h-BN Substrate } & \multicolumn{2}{|c|}{$\mathrm{SiO}_{2}$ Substrate } \\
\hline & Values & Ref. & Values & Ref. \\
\hline Channel Length $(L(\mu m))$ & 1 & [22] & 5 & [14] \\
\hline Channel Width $(W(\mu m))$ & 1 & [22] & 25 & [14] \\
\hline Top Gate Oxide & & & & \\
\hline Thickness $\left(t_{o x}(n m)\right)$ & 8.5 & {$[22]$} & 15 & {$[14]$} \\
\hline Bottom Gate Oxide & & & & \\
\hline thickness $\left(t_{b o x}(n m)\right)$ & - & - & 300 & {$[14]$} \\
\hline Dielectric Constant of & & & & \\
\hline $\begin{array}{l}\text { Top Gate }\left(\epsilon_{o x}(F / m)\right) \\
\text { Dielectric Constant of }\end{array}$ & 3.9 & {$[22]$} & 8.9 & {$[14]$} \\
\hline $\operatorname{Bottom}\left(\epsilon_{b o x}(F / m)\right)$ & - & - & 3.9 & {$[14]$} \\
\hline $\begin{array}{l}\text { Dielectric Constant of } \\
\text { vacuum }\left(\epsilon_{0}(F / m)\right)\end{array}$ & $8.85 \times 10^{12}$ & [22] & $8.85 \times 10^{12}$ & [14] \\
\hline Temperature $(T(K))$ & 300 & {$[22]$} & 300 & {$[14]$} \\
\hline Fermi velocity $\left(v_{f}(c s / s e c)\right)$ & $2-3 \times 10^{8}$ & {$[22]$} & $2-3 \times 10^{8}$ & {$[14]$} \\
\hline $\begin{array}{l}\text { Surface Phonon energy } \\
\text { of substrate }(\hbar \omega(m e V)) \\
\text { Graphene/Substrate Interface }\end{array}$ & 200 & {$[28]$} & 56 & {$[29]$} \\
\hline $\begin{array}{l}\text { Graphene/substrate Interrace } \\
\text { Trap Density }\left(N_{i m p}\left(\mathrm{~cm}^{-2}\right)\right) \\
\text { Deformation potential of }\end{array}$ & $2.5 \times 10^{10}$ & {$[24]$} & $2.7 \times 10^{11}$ & {$[24]$} \\
\hline $\begin{array}{l}\text { Acoustic Phonons }\left(D_{A P}(e V)\right) \\
\text { Deformation energy of }\end{array}$ & 18 & [10] & 18 & {$[10]$} \\
\hline Optical Phonons $\left(D_{O P}(\mathrm{eV} / \mathrm{cm})\right)$ & $10^{6}$ to $1.29 \times 10^{9}$ & {$[10]$} & $5.14 \times 10^{7} \pm 10$ & {$[10]$} \\
\hline
\end{tabular}

We validate our proposed model for backscattering coefficient and mobility by substituting it into the drain current equation. The model simulation is performed by numerical computing environment MATLAB [21]. The simulation results are compared with experimental data extracted from [13],[23], where GFET device having $\mathrm{SiO}_{2}$ and h-BN substrate respectively. Based on these observations, $\mathrm{h}-\mathrm{BN}$ is a suitable substrate of reliable graphene devices for Analog/RF applications.

\section{Device Fabrication}

The graphene-on-BN structure shown in Figure 1(a), is fabricated by mechanical transfer process, where h-BN layers are exfoliated from Pristine h-BN single crystals and transferred onto predefined metal gates $(1 \mathrm{~nm} \mathrm{Cr} / 20 \mathrm{~nm}$ $\mathrm{AuPd})$. The device utilizes the same h-BN dielectric layer as both a supporting substrate and local-gate dielectric [22]. While in graphene-on- structure, the graphene monolayer sheet is grown by chemical vapour deposition (CVD) on copper substrates. After the growth, polymethyl methacrylate (PMMA) is coated on the graphene films, and the copper substrates are etched away in 
(a)

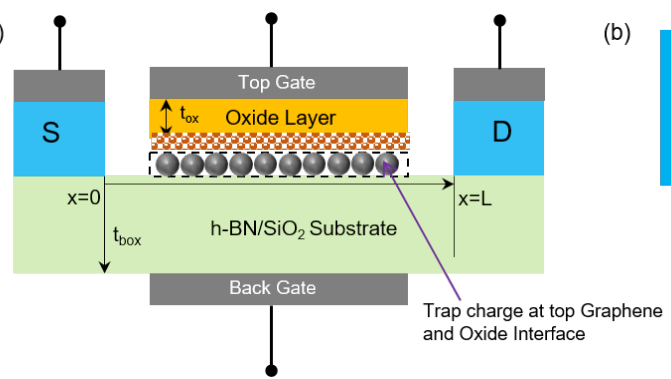

(b)

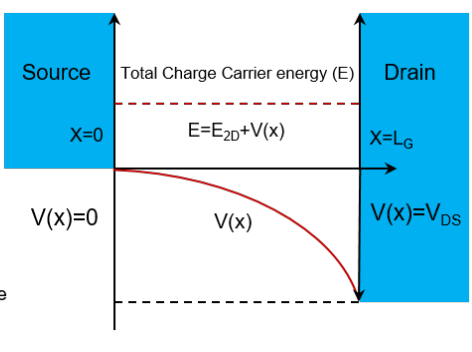

Fig. 1: (a) Crossectional view of modeled Graphene-on-h-BN and $\mathrm{SiO}_{2}$ substrate, (b) 1-D diagram of the energy of the charge carrier in channel region of GFET device.

a copper etchant and diluted $\mathrm{HCl}$. The graphene sheets are then transferred onto polished Si wafers with 300-nm thermally grown on top [23].

\section{Model Development}

\subsection{Backscattering Coefficient}

The backscattering coefficient $(R)$ is the ratio between fluxes originated from source terminal and returning back to it after scattering. Here, the different scattering mechanisms are carefully consider during the formulation of R. Now the $2 \mathrm{D}$ backscattering mean path can be given as [20]

$$
\lambda_{2 D}(x)=v_{x} \tau_{2 D}
$$

where $v_{x}=(2 / \pi) v_{f}$ is the average velocity of the charge carriers, $\tau_{2 D}$ is the electron-phonon scattering rates is given by

$$
\frac{1}{\tau_{2 D}}=\frac{1}{\tau_{A P}}+\frac{1}{\tau_{O P}}+\frac{1}{\tau_{C I}}
$$

where, $\tau_{A P}, \tau_{O P}$, and $\tau_{C I}$ are the acoustic phonon, optical phonon and charge impurity scattering rates respectively. The AP scattering rate is $[10]$

$$
\frac{1}{\tau_{A P}}=\frac{1}{\hbar^{3}} \frac{D_{A P}^{2} k_{B} T}{4 v_{f}^{2} \rho_{m} v_{p h}^{2}} E
$$

The OP scattering rate is

$$
\frac{1}{\tau_{O P}}=\frac{D_{O P}^{2}}{2 \hbar \rho_{m}\left(\hbar \omega_{o p}\right) v_{f}^{2}}\left[E-\hbar \omega_{O P}\right]
$$


The charge impurity scattering rate is described as,

$$
\frac{1}{\tau_{C I}}=\left(\frac{h v_{f}^{2}}{20 E}\right) N_{i m p}
$$

where $\hbar=1.05 \times 10^{-34} \mathrm{~m}^{2} \mathrm{~kg} / \mathrm{sec}$ is the reduced Plank's constant, $k_{B}$ is the Boltzmann constant, $T=300 \mathrm{~K}$ is the room temperature, $v_{f}$ is the Fermi velocity, $\rho_{m}=7.6 \times 10^{-7} \mathrm{~kg} / \mathrm{m}^{2}$ is the mass density, $v_{p h}=2 \times 10^{4} \mathrm{~m} / \mathrm{sec}$ is the AP velocity of charge carrier in graphene, $h=6.62 \times 10^{-34} \mathrm{~m}^{2} \mathrm{~kg} / \mathrm{sec}$ is the Plank's constant, $N_{i m p}$ is the charge carrier impurity density at graphene/substrate interface [24], $\hbar \omega_{O P}$ is the optical phonon energy, the surface OP energies of $\mathrm{SiO}_{2}$, and h-BN substrates, $D_{A P}$ is the deformation potential of acoustic phonons, $D_{O P}$ is the deformation energy of optical phonons, this energy for $\mathrm{SiO}_{2}$, and h-BN are $5.14 \times 10^{7}$, and $10^{6}$ to $1.29 \times 10^{9} \mathrm{eV} / \mathrm{cm}$ respectively [10]. $E$ is the energy of the charge carrier in channel region of GFET, is given by following the Beranger and Wilkins (see Figure 1(b)) [25],[26]

$$
E=E_{2 D}+q V(x)
$$

where, $V(x)=x E_{x}$ is the channel potential along (x-axis) the channel length, $E_{x}$ is the constant electric field in x-direction, and $E_{2 D}$ is the charge carrier average energy, which can be written as,

$$
E_{2 D}=2 k_{B} T\left[\frac{\Im_{2}\left(\eta_{f}\right)}{\Im_{1}\left(\eta_{f}\right)}\right]
$$

where $\Im_{i}\left(\eta_{f}\right)$ is the Fermi-Dirac integral of $i^{t h}$ order, $\eta_{f}=E_{f} / k_{B} T$, and $E_{f}=\hbar v_{f} \sqrt{\pi n_{2 D}}$ is the Fermi energy, and $n_{2 D}$ is impurity carrier density in graphene monolayer.

One flux method is used to relate the positive and negative flux, is given by $[17],[18]$

$$
\frac{d \vec{n}(x) \vec{v}(x)}{d x}=-\frac{\vec{n}(x) \vec{v}(x)}{\lambda_{2 D}(x)}+\frac{\overleftarrow{n}(x) \overleftarrow{v}(x)}{\lambda_{2 D}(x)}
$$

where $\vec{n}(x)$ and $\overleftarrow{n}(x)$ is the positive and negative moving charge carriers respectively. Here it is assumed that the velocity of positive and negative charge carriers are same (i.e. $\vec{v}(x)=\vec{v}(x)=v(0)$ ), so the equation (8) will become

$$
\frac{d \vec{n}(x) \vec{v}(x)}{d x}=-\frac{(\vec{n}(x)-\overleftarrow{n}(x)) v(0)}{\lambda_{2 D}(x)}
$$

Using the boundary condition $\overleftarrow{n}(x)=0$ and the current continuity equation $\vec{n}(x) v(L)=$ and integrating equation (9) with respect to $x$ we have

$$
\int_{0}^{L} \vec{v}(x) d \vec{n}(x)=\int_{0}^{L}-\frac{-(\vec{n}(0)-\overleftarrow{n}(0))}{\lambda_{2 D}(x)} d x
$$


Now substituting the value of $\lambda_{2 D}(x)$ from equation (1) and solving the equation (10) using the boundary conditions

$$
\overleftarrow{n}(0) v(0)=[-(\vec{n}(0)-\overleftarrow{n}(0)) v(0)] \times \frac{2}{\pi v_{f}}\left[\frac{\aleph}{q E} \ln \left[E_{2 D}+q E_{x}\right]\right]_{0}^{L}+\Re\left[E_{2 D} x+q E \frac{x^{2}}{2}\right]_{0}^{L}
$$

Now the expression for backscattering coefficient is the ratio of positive and negative going flux is given by,

$$
R=\frac{2\left[\frac{\aleph}{q E_{x}} \ln \left[1+\frac{L}{L_{K T}}\right]\right]+\Re L E_{2 D}\left[1+\frac{L}{L_{K T}}\right]}{\pi v_{f}+2\left[\frac{\aleph}{q E_{x}} \ln \left[1+\frac{L}{L_{K T}}\right]\right]+{ }_{2 D}\left[1+\frac{L}{L_{K T}}\right]}
$$

where, $\aleph=\frac{h v_{f}}{20} N_{i m p}, \Re=A+B, A=\frac{1}{\hbar^{3}} \frac{D_{A P}^{2} k_{B} T}{4 v_{f}^{2} \rho_{m} v_{p h}^{2}}, B=\frac{D_{O P}^{2}}{2 \hbar \rho_{m}\left(\hbar \omega_{O P}\right) v_{f}^{2}}$, and $L_{K T}=\frac{E_{2 D}}{q E_{x}}$ is the distance at which the potential drops to the value $\left(k_{B} T\right) / q$, and $E_{2 D}$ is the average energy of the charge particles.

\subsection{Quasi-Ballistic Mobility}

The field dependant expression of quasi-ballistic $\left(\mu_{e f f}\right)$ model for 2D GFET is given by [20];

$$
\mu_{e f f}=\frac{2 q T\left(\left\langle E_{2 D}\right\rangle\right) M\left(E_{F}\right)}{N_{2 D}}
$$

where, $\mathrm{h}$ is the Plank's constant, $T\left(\left\langle E_{2 D}\right\rangle\right)$ is the transmission coefficient, and $M\left(E_{F}\right)$ is the number of channel, which is given by,

$$
M(E)=\frac{2 W E}{\pi \hbar v_{f}}
$$

where, $W$ is the width of the graphene monolayer, $E=\hbar v_{f} k$ is the band energy. The transmission coefficient $T\left(\left\langle E_{2 D}\right\rangle\right)$ is [27]

$$
T\left(\left\langle E_{2 D}\right\rangle\right)=1-R\left(\left\langle E_{2 D}\right\rangle\right)
$$

From (12), we see that backscattering coefficient is dependent on the carrier energy mean free path via different scattering mechanisms.

Now the expression for the quasi-ballistic is,

$$
\mu_{e f f}=\frac{2 q}{h} \frac{T\left(\left\langle E_{2 D}\right\rangle\right) M\left(E_{F}\right)}{N_{2 D}}
$$

or,

$$
\mu_{e f f}=\frac{4 q}{\pi h^{2} v_{f}} \frac{2 W E}{N_{2 D}}[1-R]
$$


where, $R$ is the backscattering coefficient given in equation (12), $N_{2 D}$ is the carrier concentration in graphene monolayer, which indicates that $\mu$ decreases with the increase in $N_{2 D}$. While remaining symbols have their usual meanings.

\section{Drain Current Calculation}

The drain current expression for graphene FET in quasi-ballistic regime using the MFT is given by [28],[29]

$$
I_{D S}=\frac{q W V_{T h, 2 D} n(x)(1-R)\left[1-\exp \left(-U_{D S}\right)\right]}{1+R+(1-R) \exp \left(U_{D S}\right)}
$$

where, $W$ is the width of the graphene monolayer, $V_{T h, 2 D}=V_{T} h \frac{\sqrt{\pi}}{2}$ is $2 \mathrm{D}$ thermal velocity, $V_{T h}=\sqrt{\left(2 k_{B} T\right) / \pi m^{*}}$ is the thermal velocity, $m^{*}$ is the effective mass of the charge carriers, $n(x)=\frac{q^{3} V_{c h} \mathrm{~V}_{c h}}{\pi\left(\hbar v_{f}\right)^{2}}$ is the sheet carrier density in graphene monolayer, $U_{D S}=\left(q V_{D S}\right) /\left(k_{B} T\right), V_{D S}$ is the drain to source voltage $R$ is the back scattering coefficient, which is defined thoroughly in the model development section, and $V_{c h}$ is the channel potentials, which is the depends on the oxide capacitances and bias voltages, is given by [30]

$$
V_{c h}(x)=\left(V_{T, e f f}-\frac{V_{D S}}{2}\right) \frac{C_{t o x}}{C_{t o x}+C_{b o x}+0.5 C_{q}}+\left(V_{B, e f f}-\frac{V_{D S}}{2}\right) \frac{C_{b o x}}{C_{t o x}+C_{b o x}+0.5 C_{q}}
$$

where, $C_{t o x}=\frac{\epsilon_{t o x}}{t_{o x}}, C_{b o x}=\frac{\epsilon_{b o x}}{t_{b o x}}$ is the capacitance per unit area formed by top, back oxide, $t_{t o x} / t_{\text {box }}$ is the thickness of top, back oxide layers and $\epsilon_{t o x} / \epsilon_{b o x}$ is permittivity of the top and back gate oxide materials. $V_{T, \text { eff }}=\left(V_{T G S}-\right.$ $\left.V_{T G S, 0}\right)$ and $V_{B, e f f}=\left(V_{B G S}-V_{T G S, 0}\right)$, where are the top and back gate overdrive voltages respectively. Theses overdrive voltages comprise of the work function difference between gate oxide and graphene channel as well as the possible additional charge density due to impurity or doping. $C_{q}$ is the quantum capacitance. The expression for quantum capacitance is [31],

$$
C_{q}=\frac{2 q^{2}}{\pi \hbar \sqrt{v_{f}}}\left[\left|N_{G}\right|+N_{i m p}\right]^{\frac{1}{2}}
$$

where,

$$
N_{G}=\left(\frac{q\left|V_{c h}\right|}{\hbar v_{f} \sqrt{\pi}}\right)^{2}
$$

where, $N_{G}$ is the gate voltage induced channel charge density, and remaining symbols have their usual meanings. 
(a)

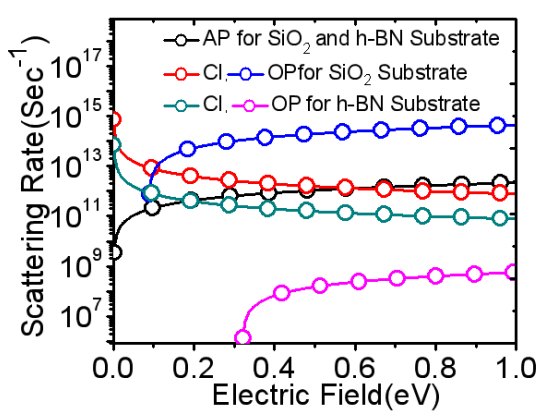

(c)

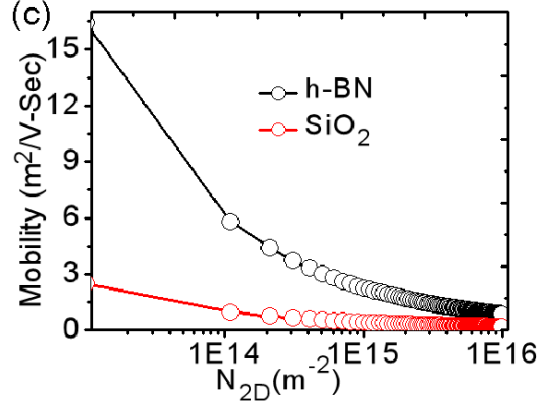

(b)

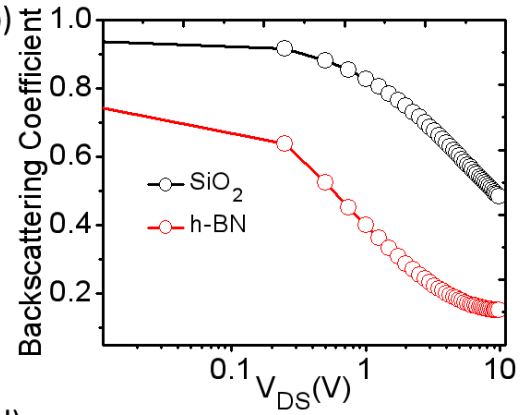

(d)

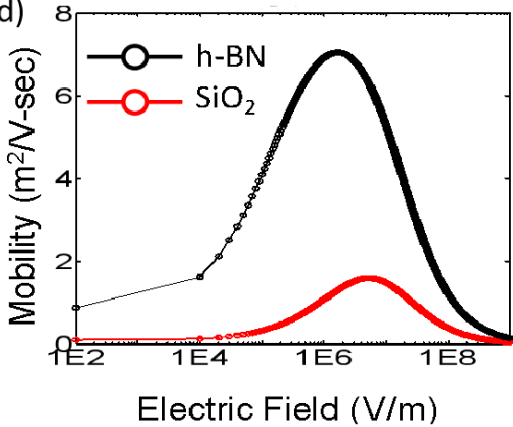

Fig. 2: (a) Acoustic phonon (AP), optical phonon (OP), and charge impurity (CI) scattering rates computed as a function of electron energy for all phonon scattering mechanisms on a $\mathrm{SiO}_{2}$ and h-BN substrate. (b) The backscattering coefficient vs drain to source voltage for $\mathrm{SiO}_{2}$ and h-BN substrates. (c) The quasi-ballistic mobility as a function of carrier density, (d) as a function of electric field.

\section{Results and Discussion}

The numerical computing software MATLAB [21] is used for the simulation of our proposed analytical compact model. Using this set of expressions in simulate the $I_{D S}$ for GFET device having $\mathrm{SiO}_{2}$ and h-BN substrates are compared with experimental data extracted from [22] and [23].

The scattering rates are the important factor, because it strongly affects the backscattering coefficient and charge carrier mobility shown in figure 2 (a). The acoustic phonon scattering plays an important role in piezoelectric substrates, is considered in the formulation of backscattering coefficient and quasi-ballistic mobility. However, the optical phonon (OP) and charge impurity (CI) scatterings mechanism are induced by the substrate of GFET device. The CI scattering is depends upon the graphene/substrate interface trap carrier density, for h-BN substrate CI scattering is ten times lower than the $\mathrm{SiO}_{2}$ substrate. The h-BN substrate has smaller backscattering coefficient as compare to the $\mathrm{SiO}_{2}$ substrate. This is happen due to lower charge trap density at graphene/substrate interface, and higher optical phonon energy. The h-BN 

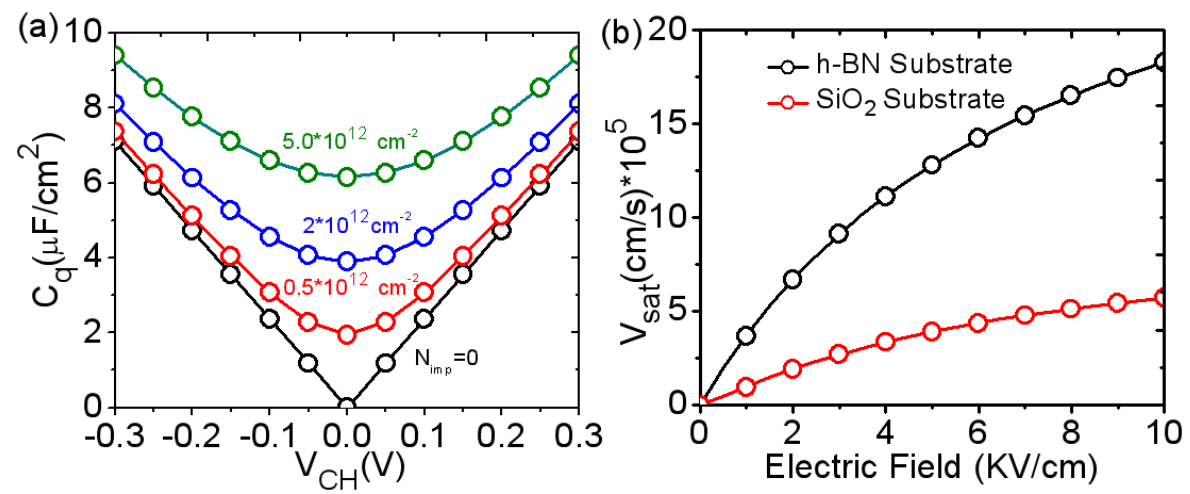

Fig. 3: (a) Quantum capacitance $\left(C_{q}\right)$ of graphene calculated against the channel voltage $\left(V_{c h}\right)$ at different $N_{i m p}$. (b) The carrier velocity vs the electric field at two different impurity densities,

and $\mathrm{SiO}_{2}$ substrates have the surface optical phonon energy of 200 and $55 \mathrm{meV}$ respectively. The high optical energy of h-BN substrate will suppress the most of the surface OP scattering. While the lower optical energy is not sufficient to suppress the surface OP scattering by the Pauli exclusion Principle [32].

The figure 2 (b) presents the backscattering coefficient $(R)$ of the charge carrier in graphene with respect to the drain to source voltage $\left(V_{D S}\right)$ for h-BN and $\mathrm{SiO}_{2}$ substrates. The backscattering coefficient in channel region is almost constant at lower values of $V_{D S}$. While at higher value of $V_{D S}, \mathrm{R}$ is deceases, i.e. the charge carrier in graphene channel is more directed at higher value of $V_{D S}$ and having less scattered with the in-plane and out-plane charge carriers.

The figure 2 (c) presents the carrier mobility in quasi-ballistic regime with respect to the drain to source voltage $\left(V_{D S}\right)$ at constant value of the electric field $\left(E_{x}\right)$ for h-BN and $\mathrm{SiO}_{2}$ substrates. The mobility of charge carrier for GFET on a $\mathrm{SiO}_{2}$ substrate decreases with respect to the $N_{2 D}$, while it has lower dependency of the carrier density as compare to GFET on h-BN substrate. The quasi-ballistic mobility of charge carrier in GFET device for both substrates is decreased due to the CI scattering mechanism. The CI scattering mechanism is strongly depends on the impurity density of the substrates. The GFET device with h-BN substrates exhibits more fluctuations in mobility, due to charged impurities that are one or two orders of magnitude lower than the $\mathrm{SiO}_{2}$ substrate. For lower value of $N_{2 D}$ the carrier mobility of GFET for h$\mathrm{BN}$ has $165000 \mathrm{~cm}^{2} / V-s$. From figure $2(\mathrm{~d})$, it appears that the mobility is increasing as a function of electric field $\left(E_{x} \approx 10^{6} \mathrm{~V} / \mathrm{m}\right)$, and decreases sharply at higher value of electric field. Because according to equation (6) the average energy of charge carrier depends upon the Fermi energy, and the high electric field may increases the scatterings among the charge carriers, this may cause of decrease in quasi-ballistic mobility of the charge carrier. After investigation of these two substrates we found that the h-BN maintains high carrier transport and is reliable substrate of graphene based FET devices for high-speed circuit 

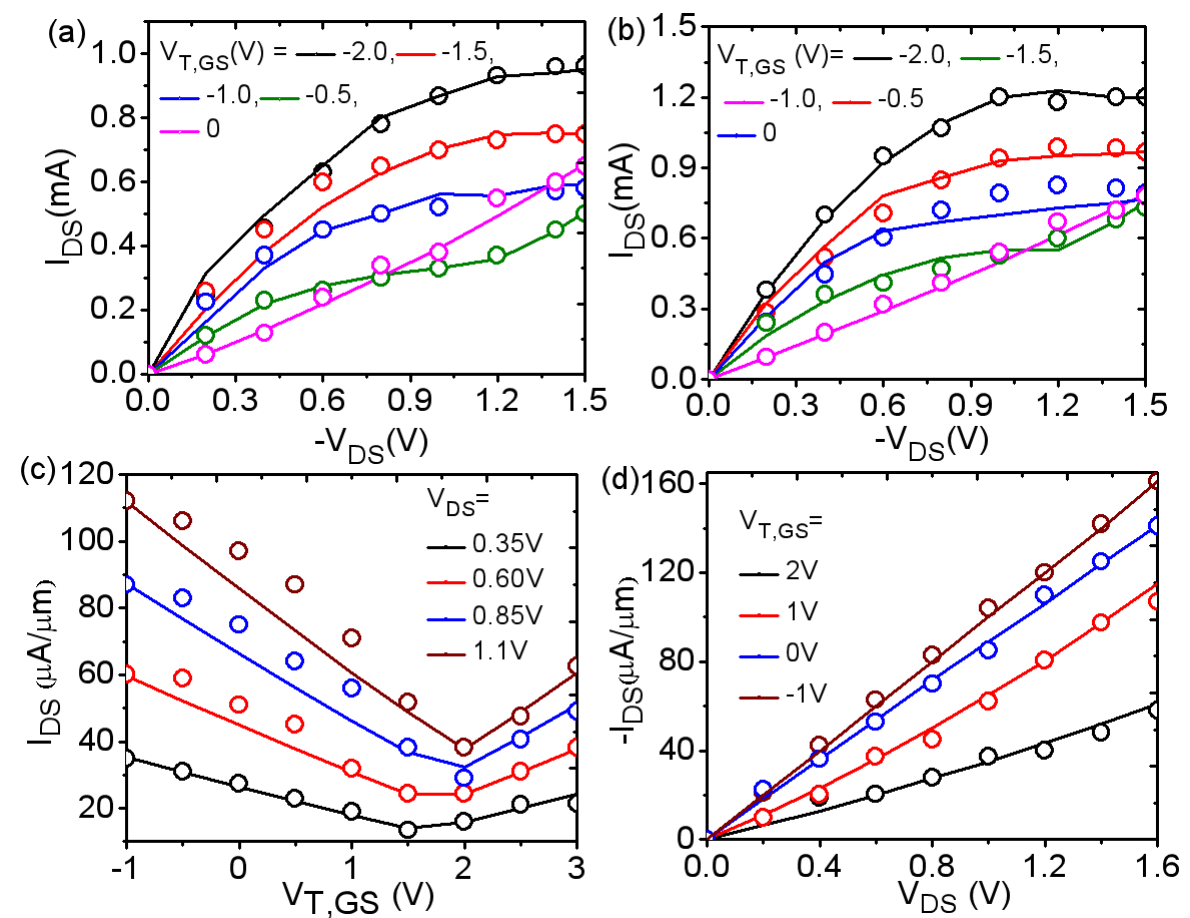

Fig. 4: The modeled output characteristics of graphene-on-h-BN and $\mathrm{SiO}_{2}$ substrates. The symbols are the experimental data taken from the [13], [14], while lines are simulation results. The output characteristics for top gated GFET (at different $V_{T, G S}$ values) (a) channel length of $1 \mu m$, (b) channel length of $440 \mathrm{~nm}$. (c) Drain current v/s gate voltage at different $V_{D S}$. (d) Drain current $\mathrm{v} / \mathrm{s}$ drain voltage with fixed $V_{B, G S}$ at $0 \mathrm{~V}$.

applications. After investigation of these two substrates we found that the h-BN maintains high carrier transport and is reliable substrate of graphene based FET devices for high-speed circuit applications.

The figure $3(\mathrm{a})$, shows the channel voltage $\left(V_{c h}\right)$ dependent quantum capacitance $\left(C_{Q}\right)$ and revealed the band symmetry of graphene material. However, $C_{Q}$ is used to measure density of states of graphene directly and also an efficient way to explore various peculiarity occurring near the Dirac point. The figure $3(\mathrm{~b})$, is the plot of the carrier velocity versus the electric field for different trap charge densities, at graphene/substrate interface. The higher trap density will increase the charged impurity scattering rate, which has a large impact on the carrier velocity. For this purpose, the GFET device having the lower trap density at graphene/h-BN substrate interface, causes higher carrier velocity.

Now using the expression of $\mathrm{R}$ from equation (12) and $\mu_{e f f}$ from equation (17) in the $I_{D S}$ equation (18), the output characteristics of GFET device having the h-BN and $\mathrm{SiO}_{2}$ substrate respectively are shown in figure 4 . The 
figure 4(a) and (b) shows the output characteristics of top-gated GFET device having $1 \mu \mathrm{m}$ and $440 \mathrm{~nm}$ gate lengths respectively. At $V_{T, G S}=-0.5 \mathrm{~V}$, the observed effect is known as 'kink' effect, which is describe the peculiar ambipolar behaviour of charge carrier in the channel region of GFET [31]. While the flat saturation observed at $V_{T, G S} \leq 1 V$. The $I_{D S}$ in figure $4(\mathrm{c})$ is obtained against $V_{T, G S}$ at different value of $V_{D S}$ increasing from 0.35 to $1.1 \mathrm{~V}$. There is a clear dependence of the minimum conduction point on $V_{D S}$. While, figure $4(\mathrm{~d})$ describes the transfer characteristics of GFET with $\mathrm{SiO}_{2}$ substrate at different value of top-gate voltage where 'kink' effect observed at $V_{D S}=-1.0 \mathrm{~V}$.

\section{Conclusion}

In this paper, we present an explicit model for backscattering coefficient and mobility of charge carrier for graphene-on-substrate (h-BN and $\mathrm{SiO}_{2}$ ) FET devices. Here the substrate induced scattering rates are carefully considered during the formulation of quasi-ballistic mobility, and observed that h-BN is most suitable material for GFET device. It also observed that at high electric field the mobility of charge carrier is greatly reduced due to scattering among the charge carriers. The proposed expressions of $R$ and $\mu$ are used in the $I_{D S}$ equation to simulate the peculiar output characteristics of GFET devices. Further from this paper it is clear that the h-BN substrate is more reliable for high performance GFET devices. It offers the higher carrier mobility and lower backscattering coefficient. The proposed expression of quasi-ballistic mobility and backscattering coefficient is used in $I_{D S}$ equation, which is simplified enough to implement in HDL (Verilog-A). The HDLs describes the behaviour of model in terms of their ports and parameters applied, and used for the circuit implementations in Cadence like circuit simulator environments.

\section{References}

[1] Wu, Y., Farmer, D. B., Xia, F., and Avouris, P., Graphene Electronics: Materials, Devices, and Circuits. Proceedings of the IEEE, 101, (7), pp. 1620-1637, (2013)

[2] Novoselov, K., Falko, V., Colombo, L. et al. A roadmap for graphene. Nature 490, 192-200 (2012)

[3] Moon, J. S., Antcliffe, M., Seo, H. C., Lin, S. C., Schmitz, A., Milosavljevic, I., McCalla, K., Wong, D., Gaskill, D. K., Campbell, P. M., Lee, K.M., and Asbeck, P., Graphene review: An emerging RF technology. 2012 IEEE 12th Topical Meeting on Silicon Monolithic Integrated Circuits in RF Systems, (2012)

[4] Schwierz, F. Graphene transistors. Nature Nanotech 5, 487-496 (2010). 
[5] Chauhan, J., and Guo, J., High-field transport and velocity saturation in graphene. Applied Physics Letters, 95, (2), 023120, (2009)

[6] Hwang, E. H. and Das, S.,, Dielectric function, screening, and plasmons in two-dimensional graphene. Physical Review B, 75, (20), (2007)

[7] Li, X., Barry, E. A., Zavada, J. M., Nardelli, M. B., and Kim, K. W., Surface polar phonon dominated electron transport in graphene. Applied Physics Letters, 97, (23), 232105, (2010)

[8] Dean, C., Young, A., Meric, I. et al. Boron nitride substrates for highquality graphene electronics. Nature Nanotech 5, 722-726 (2010)

[9] Bidmeshkipour, S., Vorobiev, A., Andersson, M. A., Kompany, A., and Stake, J., Effect of ferroelectric substrate on carrier mobility in graphene field-effect transistors. Applied Physics Letters, 107, (17), 173106, (2015)

[10] Hirai, H., Tsuchiya, H., Kamakura, Y., Mori, N., and Ogawa, M., Electron mobility calculation for graphene on substrates. Journal of Applied Physics, 116, (8), 083703, (2014)

[11] Tiwari, D. L., and Sivasankaran, K., Impact of substrate on performance of band gap engineered graphene field effect transistor. Superlattices and Microstructures, 113, 244-254, (2018)

[12] Haddad, P.A. Haddad, Flandre, D. Flandre, and Raskin, J.P. Raskin, A Quasi-Static Model of Silicon Substrate Effects in Graphene Field Effect Transistors IEEE Electron Device Letters, 38, (7), 987-990, (2017)

[13] Meric, I., Han, M. Y., Young, A. F., Ozyilmaz, B., Kim, P., and Shepard, K. L., Current saturation in zero-bandgap, top-gated graphene field-effect transistors. Nature Nanotechnology, 3, (11), 654-659, (2008)

[14] Dorgan, V. E., Bae, M.H., and Pop, E., Mobility and saturation velocity in graphene on $\mathrm{SiO}_{2}$. Applied Physics Letters, 97, (8), 082112, (2010)

[15] Zhu, W. Zhu, Perebeinos, V. Perebeinos, Freitag, M. Freitag, and Avouris, P. Avouris, Carrier scattering, mobilities, and electrostatic potential in monolayer, bilayer, and trilayer graphene. Physical Review B, 80, 23, (2009)

[16] Xue, J., Yamagishi, J. S., Bulmash, D., Jacquod, P., Deshpande, A., Watanabe, K., Taniguchi, T., Herrero, P. J., and LeRoy, B. J., Scanning tunnelling microscopy and spectroscopy of ultra-flat graphene on hexagonal boron nitride. Nature Materials, 10, (4), 282-285, (2011)

[17] Clerc, R., Palestri, P., and Selmi, L., On the physical understanding of the kT-layer concept in quasi-ballistic regime of transport in nanoscale devices. 
IEEE Transactions on Electron Devices, 53, (7), 1634-1640, (2006)

[18] Kim, R. and Lundstrom, M. S., Physics of Carrier Backscattering in One- and Two-Dimensional Nanotransistors. IEEE Transactions on Electron Devices, 56, (1), 132-139, (2009)

[19] McKelvey, J. P., Longini, R. L., and Brody, T. P., Alternative Approach to the Solution of Added Carrier Transport Problems in Semiconductors. Physical Review, 123, (1), 51-57, (1961)

[20] Verma, R., Bhattacharya, S., and Mahapatra, S., Modeling of Temperature and Field-Dependent Electron Mobility in a Single-Layer Graphene Sheet. IEEE Transactions on Electron Devices, 60, (8), 2695-2698, (2013)

[21] "MATLAB," MathWorks, Inc. (2010) [Online]. Available:http://www.mathworks. com/ products/Matlab.

[22] Meric, I., Dean, C., Young, A., Hone, J., Kim, P., and Shepard, K. L., Graphene field-effect transistors based on boron nitride gate dielectrics. 2010 International Electron Devices Meeting, (2010).

[23] Wang, H., Hsu, A., Kong, J., Antoniadis, D. A., and Palacios, T., Compact Virtual-Source Current-Voltage Model for Top- and Back-Gated Graphene Field-Effect Transistors. IEEE Transactions on Electron Devices, 58, (5), 1523-1533, (2011)

[24] Burson, K. M., Cullen, W. G., Adam, S., Dean, C. R., Watanabe, K., Taniguchi, T., Kim, P., and Fuhrer, M. S., Direct Imaging of Charged Impurity Density in Common Graphene Substrates. Nano Letters, 13, (8), 3576-3580, (2013)

[25] Zhu, W., Perebeinos, V., Freitag, M., and Avouris, P., Carrier scattering, mobilities, and electrostatic potential in monolayer, bilayer, and trilayer graphene. Physical Review B, 80, (23), (2009)

[26] Baranger, H. U. and Wilkins, J. W., Ballistic electrons in an inhomogeneous submicron structure: Thermal and contact effects. Physical Review B, 30, (12), 7349-7351, (1984).

[27] Chen, M. J., Huang, H. T., Chou, Y.C., Chen, R. T., Tseng, Y. T., Chen, P. N., and Diaz, C. H., Separation of Channel Backscattering Coefficients in Nanoscale MOSFETs. IEEE Transactions on Electron Devices, 51, (9), 1409-1415, (2004)

[28] Upadhyay, A. K., Kushwaha, A. K., and Vishvakarma, S. K., A Unified Scalable Quasi-Ballistic Transport Model of GFET for Circuit Simulations. IEEE Transactions on Electron Devices, 65, (2), 739-746, (2018) 
[29] Upadhyay, A. K., Kushwaha, A. K., Rastogi, P., Chauhan, Y. S., and Vishvakarma, S. K., Explicit Model of Channel Charge, Backscattering, and Mobility for Graphene FET in Quasi-Ballistic Regime. IEEE Transactions on Electron Devices, 65, (12), 5468-5474, (2018)

[30] Upadhyay, A. K. Upadhyay, Kushwaha, A. K. Kushwaha, Gupta, D. Gupta and Vishvakarma, S. K. Vishvakarma, Scalable Electrical Compact Modeling for Graphene FET Transistors. IEEE Transactions on Nanotechnology, 12, (4), 539-546, (2013).

[31] Xia, J. Xia, Chen, F. Chen,Li, J. Li, and Tao, N. Tao, Measurement of the quantum capacitance of graphene. Nature Nanotechnology, 4, (8), 505-509, (2009)

[32] David, J. K., Register, L. F., and Banerjee, S. K., Semiclassical Monte Carlo Analysis of Graphene FETs. IEEE Transactions on Electron Devices, 59, (4), 976-982, (2012) 